\title{
Strategic Diversification for Asynchronous Asset Trading: Insights from Generalized Coherence Analysis of Cryptocurrency Price Movements
}

\author{
Nirvik Sinha, ${ }^{* \dagger}$ Yuan Yang
}

\begin{abstract}
Non-linear interactions between cryptocurrency price movements can elicit crossfrequency coupling $(\mathrm{CFC})$ wherein one set of frequencies in the $1^{\text {st }}$ timeseries is coupled to another set of frequencies in the $2^{\text {nd }}$ timeseries. To investigate this, we use a generalized coherence approach to detect and quantify both linear (i.e., iso-frequency coupling, IFC) and non-linear coherence (CFC) and the associated phase relationships between the intra-day price changes of various pairs of cryptocurrencies for the year 2020. Using this information, we further assess the risk reduction associated with diversification of portfolios between each pair of a small market capital and a large market capital cryptocurrency, for both synchronous and asynchronous trading conditions. While mean pairwise IFC values were lower for smaller cryptocurrencies, pairwise CFC values were more heterogeneous and had no correlation with the market capital size. Diversification of portfolios resulted in reduced risk for synchronously-traded pairs of those cryptocurrencies which had low IFC. For asynchronous trading conditions, if the larger market capital cryptocurrency was traded at a higher frequency, diversification almost always reduced risk. Thus, the novel approach used in this study reveals important insights into the complex dynamics that govern the price trends of cryptocurrencies.
\end{abstract}

\section{Introduction}

Since its inception in $2008,{ }^{1}$ cryptocurrencies have been increasingly drawing the attention of investors and the general public for good and bad reasons alike. They have been hailed for their highly secure blockchain technology facilitating fast, trustless and borderless financial transactions, fostering healthy economic competition and lowering market entry barriers. ${ }^{2}$ Furthermore, the year 2020 has seen the emergence of decentralized financing in the

\section{*0x481d4E56Ba29deD0aCEDA11a293d5D6E0cB58801}

†Nirvik Sinha (nirviksinha2024@u.northwestern.edu) is a PhD candidate in Interdepartmental Neuroscience at Feinberg School of Medicine, Northwestern University, IL, USA.

tYuan Yang (yan.yang-2@ou.edu) is an assistant professor at Stephenson School of Biomedical Engineering, University of Oklahoma, Tulsa, OK, USA. 
cryptocurrency sphere. It may potentially revolutionize the traditional financial system by broadening financial inclusion, promoting barrier-free access and permissionless innovation, thereby heralding new opportunities for entrepreneurs and innovators. ${ }^{3}$ On the other hand, those with a more critical perspective have cautioned against several flaws associated with cryptocurrencies. For example, issues such as vulnerability to trust abuse, ${ }^{4}$ long-term sustainability of a deflationary environment created by a limited supply, ${ }^{5}$ threats from potentially devastating security flaws, and rampant misuse of privacy coins by malevolent and dishonest actors have cast doubts on the widespread acceptance of this relatively nascent technology. ${ }^{6,7}$ Moreover, a major cause for concern among potential investors is the extreme price volatility that is a hallmark of cryptocurrencies fueled by speculative investment, ${ }^{5,8}$ and occurrence of multiple price bubbles. ${ }^{9}$

Notwithstanding the mixed reaction of the general public, cryptocurrency market capitalization has seen a dramatic resurgence since its collapse in 2018. At the time of writing of this paper, the total market capital is more than 900 billion US dollars with 1300 cryptocurrencies having an individual market capital of more than 1 million US dollars. ${ }^{10}$ Despite this diversity of options, the top five cryptocurrencies still wield an enormous $86 \%$ of the total market capital. ${ }^{10}$ Such huge capital concentration, especially in Bitcoin, leads the price movements of many cryptocurrencies to be highly correlated with the ones at the top of the market capital rankings. ${ }^{11}$ It has also been shown that collaborative development of multiple cryptocurrencies leads to their correlated returns. ${ }^{12}$ Moreover, within each cluster of correlated cryptocurrencies, newer ones appear poised to follow the trend of their 'elders'. ${ }^{13}$ At times of uncertainty, herding behavior dominates the cryptocurrency market which in turn increases systematic risk and leads to loss of market efficiency. ${ }^{14}$ Nevertheless, as the cryptocurrency market continues to mature, there has been an emergence of complexity in the form of distinct assets with unique use cases. ${ }^{15}$ Along with this, unfortunately, there has also been an explosion of scams and clones of existing assets with no inherent use value. ${ }^{16}$

In such a rapidly evolving market, portfolio diversification can serve to increase returns for institutional and retail investors while insulating them from the risks of concurrent price collapses of multiple assets and fraudulent exit scams. ${ }^{17}$ Analysis of the relationship among price movements of different assets can reveal important insights into the collective behavior of the market, thereby providing an optimal means of portfolio diversification. ${ }^{18,19}$ For example, application of diagonal and asymmetric diagonal BEKK models to the intra-day price data of eight cryptocurrencies showed strong pairwise positive correlations. ${ }^{20}$ Subsequently, the bivariate diagonal BEKK model gave evidence of bi-directional price-shock transmission between Bitcoin and both Ethereum and Litecoin, and price volatility spillover effects between all three of these pairs. ${ }^{21}$ Understanding the interactions among different cryptocurrency has emerged as a powerful aid to effective and appropriate decision making for a diverse range of investment goals. ${ }^{22}$ Non-trivial hierarchical structures and cryptocurrency groupings have been found using cross-correlation analysis. Furthermore, minimum spanning trees constructed from the cross-correlation data revealed distinct and stable community structures. ${ }^{15}$

Macroeconomic timeseries are influenced by factors operating at various timescales; therefore, their relationships are not necessarily homogenous across different frequencies. ${ }^{23}$ This necessitates the use of coherence analysis for better understanding the relationships. For example, using wavelet-based coherence analysis, it was shown that specifically 8-32 day periodic correlations between online factors (e.g., Google trends, Wikipedia views, etc.) and

\section{3}


price strengthen significantly during bubble-like regimes of the cryptocurrency market. ${ }^{24}$ Applying the same approach to the top seven cryptocurrencies by market capital, another study showed that the benefit of portfolio diversification is confined to intra-week to monthly periods of trading for specific pairs. ${ }^{25}$ However, conventional coherence analysis can only detect linear interactions between two timeseries i.e., coupling at the same frequency (or scale), also known as iso-frequency coupling. Non-linear interactions, on the other hand, can elicit cross-frequency coupling wherein one set of frequencies in the $1^{\text {st }}$ timeseries is coupled to another set of frequencies in the $2^{\text {nd }}$ timeseries. For example, if the price movements of two cryptocurrencies $\mathrm{A}$ and $\mathrm{B}$ exhibit non-linear interactions then there may exist a strong correlation between the daily price movements of $\mathrm{A}$ and the hourly price movements of $\mathrm{B}$. This constitutes a harmonic interaction (wherein price movement of $A$ at 1 cycle per day is coupled to that of $B$ at 24 cycles per day). Likewise, there may also be subharmonic interactions. Therefore, assessing both the linear and non-linear interactions between cryptocurrency price movements can give a comprehensive picture of the complex dynamics that govern their market trends. Moreover, this information can be used to design risk-minimizing portfolios for those investors who trade multiple cryptocurrencies at different frequencies.

The objective of this study was to detect and quantify linear and non-linear coherence and the associated phase relationships between the intra-day price changes of various cryptocurrency pairs for the year 2020 using a generalized coherence approach. Furthermore, this approach was also used to assess the risk reduction associated with diversification of portfolios between each pair of a small market capital and a large market capital cryptocurrency, for both synchronous and asynchronous trading conditions (i.e., same and different frequency of trading for each cryptocurrency in the pair).

\section{Methods}

The list of the top 250 cryptocurrencies by market capital (as on 1 January 2021) was obtained from CoinGecko. ${ }^{26}$ All fiat currency-pegged stablecoins (e.g., USDC) were excluded from analysis. Subsequently, minutely closing price data (in United States Dollars) were obtained for all cryptocurrencies in this list that were available for the full period of 1 January to 31 December 2020, on the Binance exchange. The Python wrapper of the Public Rest API for Binance was used for this purpose. ${ }^{27}$ The Binance exchange was chosen because it is a trusted and popular cryptocurrency exchange with the highest 24-hour trading volume worldwide. ${ }^{28}$ Additionally, it also provides full and free access to data of the granularity level (i.e., minutely) used in this study.

The minutely return (i.e., price change) of each cryptocurrency was computed as:

$P_{i}(t)=\ln C_{i}(t+\delta t)-\ln C_{i}(t)$

where $C_{i}(t)$ and $P_{i}(t)$ are the price and minutely return (in USD Tether) of the $i^{t h}$ cryptocurrency, $t$ is the time and $\delta t$ is the sampling interval (=1 minute). In order to facilitate comparison between cryptocurrencies with different price volatilities, the computed returns were normalized using the following formula:

$N_{i}(t)=\frac{P_{i}(t)-\mu_{i}}{\sigma_{i}}$ 
where $N_{i}$ is the risk (i.e., volatility) -adjusted return of the $i^{t h}$ cryptocurrency. $\mu_{i}$ and $\sigma_{i}$ are the mean and standard deviation of $P_{i}$ over the entire period of study. ${ }^{15}$

For the purpose of subsequent analysis, the year-long timeseries of each cryptocurrency was segmented into 1-day non-overlapping epochs. The linear and non-linear interactions in the frequency range of 1-24 cycles per day (CPD) were assessed at the interval of $1 \mathrm{CPD}$. The generalized coherence measure, i.e., n:m coherence (NMC), was used for this purpose. ${ }^{29} \mathrm{In}$ order to facilitate the understanding of this measure, let's begin by revisiting classical coherence. Let $X(f), Y(f)$ be the Fourier Transform of the two timeseries of a pair of cryptocurrencies. The cross- $\left(S_{X Y}\right)$ and auto-spectra $\left(\mathrm{S}_{\mathrm{X}}\right.$ and $\left.S_{Y}\right)$ of the timeseries are defined as:

$S_{X Y}\left(f_{X}, f_{Y}\right)=<\mathrm{X}\left(f_{X}\right)\left(Y\left(f_{Y}\right)\right)^{*}>$

$\mathrm{S}_{\mathrm{X}}\left(f_{X}\right)=<X\left(f_{X}\right)\left(\mathrm{X}\left(f_{X}\right)\right)^{*}>$

$\mathrm{S}_{\mathrm{Y}}\left(f_{\mathrm{Y}}\right)=<Y\left(f_{\mathrm{Y}}\right)\left(\mathrm{Y}\left(f_{\mathrm{Y}}\right)\right)^{*}>$

where $(.)^{*}$ is the complex conjugate and $\langle$.$\rangle is the mean across the total number of epochs.$ The classical coherence between the two timeseries can then be computed as:

classical coherence $\left(f_{X}, f_{Y}\right)=\frac{\left|S_{X Y}\left(f_{X}, f_{Y}\right)\right|^{2}}{\mathrm{~S}_{\mathrm{X}}\left(f_{X}\right) \mathrm{S}_{\mathrm{Y}}\left(f_{Y}\right)}$

Therefore, the classical coherence is essentially the squared magnitude of the cross-spectrum $\left(S_{X Y}\right)$ normalized by the product of the auto-spectra of the individual timeseries $\left(\mathrm{S}_{X}\right.$ and $\left.S_{Y}\right)$. It provides the strength of correlation between the timeseries at different frequencies. The design of the classical coherence measure, however, constrains it to assess correlation between the two timeseries only for iso-frequency pairs where $f_{X}=f_{Y}$ i.e., linear interactions.

Based on high-order statistics, ${ }^{30}$ the concepts of cross- and auto-spectra can be extended to also include the relationship between pairs of unequal frequencies $\left(f_{X} \neq f_{Y}\right)$. For example, consider the simple case wherein we wish to explore the covariance between $X(f)$ at frequency $f_{X}$ and $Y(f)$ at frequency $2 f_{X}$. From the definition of bispectrum (see Table 3 in Nikias and Mendel, "Signal Processing"), ${ }^{30}$ the cross-spectrum for this interaction can be computed as:

$S_{X Y}^{12}\left(f_{X}, f_{X}+f_{X}\right)=<X\left(f_{X}\right) X\left(f_{X}\right)\left(\mathrm{Y}\left(f_{X}+f_{X}\right)\right)^{*}>$

which reduces to:

$S_{X Y}^{12}\left(f_{X}, 2 f_{X}\right)=<X^{2}\left(f_{X}\right)\left(\mathrm{Y}\left(2 f_{X}\right)\right)^{*}>$

Likewise, using the definition of trispectrum, one can arrive at the following formula for $1: 3$ interaction $\left(f_{Y}=3 f_{X}\right)$ :

$S_{X Y}^{13}\left(f_{X}, 3 f_{X}\right)=<X^{3}\left(f_{X}\right)\left(\mathrm{Y}\left(3 f_{X}\right)\right)^{*}>$

In general, cross- and auto-spectral interactions between a pair of unequal frequencies are given by the following equations: 


$$
\begin{aligned}
& S_{X Y}^{n m}\left(f_{X}, f_{Y}\right)=<X^{n}\left(f_{X}\right)\left(Y^{m}\left(f_{Y}\right)\right)^{*}> \\
& S_{X}^{n}\left(f_{X}\right)=<X^{n}\left(f_{X}\right)\left(X^{n}\left(f_{X}\right)\right)^{*}> \\
& S_{Y}^{m}\left(f_{X}\right)=<Y^{m}\left(f_{Y}\right)\left(Y^{m}\left(f_{Y}\right)\right)^{*}>
\end{aligned}
$$

where $S_{X Y}^{n m}$ is the higher order cross-spectrum and, $S_{X}^{n}$ and $S_{Y}^{m}$ are the higher order auto-spectra for the two timeseries. A close inspection of the equations 7-9 show that their overall form remains the same as in equations 3-5, only now, the Fourier transforms are raised to integer powers $m$ and $n$. For example,

$\mathrm{X}^{n}=\underbrace{X\left(f_{X}\right) \cdot X\left(f_{X}\right) \cdot \ldots \cdot X\left(f_{X}\right)}_{n}$.

Here, $\frac{m}{n}$ is the simple whole number ratio of $f_{X} / f_{Y}\left(e . g\right.$., if $f_{X}=8, f_{Y}=16$ then $\mathrm{m}=1, \mathrm{n}=$ 2). Using the generalized forms of the cross- and auto-spectra, one can then compute the $n: m$ coherence measure as:

$n: m$ coherence $\left(f_{X}, f_{Y}\right)=\frac{\left|s_{X Y}^{n m}\left(f_{X}, f_{Y}\right)\right|^{2}}{s_{X}^{n}\left(f_{X}\right) S_{Y}^{m}\left(f_{Y}\right)}$

Thus, the n:m coherence is a straightforward extension of the classical coherence for distinguishably determining the strength of both cross-frequency $\left(f_{X} \neq f_{Y}\right)$ and iso-frequency $\left(f_{X}=f_{Y}\right)$ coupling between the timeseries. When $f_{X}=f_{Y}$, we have $\mathrm{m}=\mathrm{n}=1$, then the NMC is equivalent to the classical (linear) coherence for iso-frequency coupling. ${ }^{31}$ When $f_{X} \neq f_{Y}$, then the NMC indicates the coupling across different frequency components (i.e., cross-frequency coupling). ${ }^{32}$ Therefore, linear (iso-frequency) as well as non-linear (cross-frequency) interactions can be assessed using this measure. The $\mathrm{n}: \mathrm{m}$ mapping can generate both integer $\left(\frac{m}{n} \in \mathbb{Z}^{+}\right)$and non-integer $\left(\frac{m}{n} \in\left(\mathbb{R}^{+}-\mathbb{Z}^{+}\right)\right)$harmonic $(\mathrm{n}>\mathrm{m})$ and sub-harmonic $(\mathrm{n}<\mathrm{m})$ coupling between the two timeseries in the frequency domain. ${ }^{29}$ As a generalized coherence method, the $\mathrm{NMC}$ is a metric indicating cross-frequency coherence between timeseries, which is different from other cross-frequency coupling methods such as the phase-amplitude coupling reflecting how a low-frequency phase modulates a high-frequency amplitude. ${ }^{33}$

According to Cauchy-Schwarz-inequality, we have:

$\left|S_{X Y}^{n m}\left(f_{X}, f_{Y}\right)\right|^{2} \leq S_{X}^{n}\left(f_{X}\right) S_{Y}^{m}\left(f_{Y}\right)$

Thus, the NMC is bounded by 0 and 1 , where 1 indicates that the two timeseries are perfectly coupled at the tested frequency pair $\left(f_{X}, f_{Y}\right)$. As the NMC values are computed by comparing different frequency pairs between timeseries, the significant threshold was adapted with a Bonferroni correction to control the type I error (family-wise error rate: 0.05 ).$^{29}$ There are 576 frequency pairs that were included for Bonferroni corrections (1-24 CPD in the $1^{\text {st }}$ timeseries $\times$ $1-24$ CPD in the $2^{\text {nd }}$ timeseries). More details of the NMC method is available in Yang et al., "A Generalized Coherence Analysis," (2016). ${ }^{29}$

The higher order phase delay $\Phi\left(f_{X}, f_{Y}\right)$ for each frequency pair $f_{X}, f_{Y}$ (where $\Phi\left(f_{X}, f_{Y}\right)=$ $n \phi\left(f_{X}\right)-m \phi\left(f_{Y}\right)$ and $\left.n f_{X}=m f_{X}\right)$ was computed as (range $=-\pi$ to $\left.+\pi\right)$ :

\section{6}


$\Phi\left(f_{X}, f_{Y}\right)=\tan ^{-1}\left\{\frac{\Re\left[S_{X Y}^{* n m}\left(f_{X}, f_{Y}\right)\right]}{\Im\left[S_{X Y}^{* n m}\left(f_{X}, f_{Y}\right)\right]}\right\}$

where the normalized higher order cross-spectrum is given by

$S_{X Y}^{* n m}\left(f_{X}, f_{Y}\right)=\frac{S_{X Y}^{n m}\left(f_{X}, f_{Y}\right)}{\sqrt{S_{X}^{n}\left(f_{X}\right) S_{Y}^{m}\left(f_{Y}\right)}}$

and $\mathfrak{R}[],. \mathfrak{I}[$.$] are the real and imaginary parts respectively. Just like the n:m coherence$ measure, equations 13-14 are straightforward extensions of the classical phase delay and normalized cross-spectrum.

Since the IFC values range from 0 to 1 and the IFC of a timeseries with itself is 1 , a distance metric $D_{i j}=1-I_{F} C_{i j}$ can be defined between every pair of cryptocurrencies $i$ and $j$ which allows the whole set of cryptocurrencies to be treated as a network. Here, each asset is a node in the network and the connectivity matrix D is a measure of adjacency between the nodes. ${ }^{34}$ An informative way of visualizing this network is to perform a multilevel hierarchical clustering of the nodes using D to construct a cluster tree or a dendrogram. In this study, the MATLAB function linkage (Statistical and Machine Learning Toolbox) was used to construct a binary cluster tree. ${ }^{35}$ In order to verify that the distance between the two clusters that contain any two assets $\mathrm{i}$ and $\mathrm{j}$ (known as the cophenetic distance) reflect the original distance $\mathrm{D}_{\mathrm{ij}}$ accurately, the correlation coefficient between them was computed (known as the cophenetic correlation coefficient) ${ }^{36}$ Subsequently, the linkage algorithm that maximized the cophenetic correlation coefficient was chosen for the cluster tree construction.

Next, the risk reduction associated with diversification of portfolio between two cryptocurrencies $(\mathrm{X}, \mathrm{Y})$ as compared to a portfolio having only the larger market capital cryptocurrency $(\mathrm{X})$ was assessed for each pair of cryptocurrencies. For this purpose, the optimal weightage of $\mathrm{Y}$ in its mixed portfolio with $\mathrm{X}$ was computed from the following formula: ${ }^{19}$

$w^{Y}=\frac{\sigma_{X}^{2}-\operatorname{cov}(X, Y)}{\sigma_{X}^{2}+\sigma_{Y}^{2}-2 \operatorname{cov}(X, Y)}$

Since we are interested in computing this measure for each trading frequency pair $\left(f_{X}, f_{Y}\right)$ individually, we used the generalized cross- and auto-spectra (which are the frequency domain analogs of $\sigma^{2}$ and $\operatorname{cov}(X, Y)$ respectively) to define a similar measure $w_{f_{X}, f_{Y}}^{Y}$ as follows:

$w_{f_{X}, f_{Y}}^{Y}=\frac{s_{X}^{n}\left(f_{X}\right)-\left|s_{X Y}^{n m}\left(f_{X}, f_{Y}\right)\right|}{s_{X}^{n}\left(f_{X}\right)+s_{Y}^{m}\left(f_{Y}\right)-2\left|s_{X Y}^{n m}\left(f_{X}, f_{Y}\right)\right|}$

where $w_{f_{X}, f_{Y}}^{X}=0$ if $w_{f_{X}, f_{Y}}^{X}<0$ and $w_{f_{X}, f_{Y}}^{X}=1$ if $w_{f_{X}, f_{Y}}^{X}>1$. The weightage of $\mathrm{X}$ is given by $w_{f_{X}, f_{Y}}^{X}=1-w_{f_{X}, f_{Y}}^{Y}$. The mixed portfolio variance is then given by:

$M_{X Y}\left(f_{X}, f_{Y}\right)=w_{f_{X}, f_{Y}}^{X} S_{X}^{n}\left(f_{X}\right)+w_{f_{X}, f_{Y}}^{Y} S_{Y}^{m}\left(f_{Y}\right)+w_{f_{X}, f_{Y}}^{X} w_{f_{X}, f_{Y}}^{Y}\left|S_{X Y}^{n m}\left(f_{X}, f_{Y}\right)\right|$

which is derived from its time domain counterpart: ${ }^{19}$ 
$\sigma_{\text {portfolio }}^{2}=w^{Y} \sigma_{Y}^{2}+w^{X} \sigma_{X}^{2}+w^{X} w^{Y} \operatorname{cov}(X, Y)$

The risk reduction of the mixed portfolio with respect to the portfolio containing only $\mathrm{X}$ for the trading frequency $f_{X}, f_{Y}$ is then defined as: ${ }^{37}$

$R R_{f_{X}, f_{Y}}=1-\frac{M_{X Y}\left(f_{X}, f_{Y}\right)}{S_{X}^{n}\left(f_{X}\right)}$

which is again analogous to its time domain counterpart:

$R R=1-\frac{\sigma_{\text {portfolio }}^{2}}{\sigma_{X}^{2}}$

Hence, the higher the value of $R R_{f_{X}, f_{Y}}$, the greater is the reduction of risk achieved by diversifying the portfolio between $\mathrm{X}$ and $\mathrm{Y}$ as compared to a portfolio containing only $\mathrm{X}$. Moreover, the risk reduction computed this way enables its categorization into (i) synchronous $\left(f_{X}=f_{Y}\right)$ and (ii) asynchronous $\left(f_{X} \neq f_{Y}\right)$ trading conditions for $\mathrm{X}$ and $\mathrm{Y}$. The second category can be further divided into two sub-categories: (i) $\left(f_{X}<f_{Y}\right)$ and (ii) $\left(f_{X}>f_{Y}\right)$. This kind of categorization is especially useful because traders often have different investment periods for different cryptocurrencies.

\section{Results}

Among the list of 250 cryptocurrencies obtained from CoinGecko, minutely trading data (in USDT) for the full period of analysis was available for 55 cryptocurrencies on Binance exchange (after exclusion of stablecoins). They are listed in Table 1.

Table 1. List of cryptocurrencies included in this study (ranked by market capitalization as of 1 January 2021). Stablecoins are excluded.

\begin{tabular}{lllll}
\hline 1. Bitcoin & 12. Theta Network & 23. Waves & 34. Hedera Hashgraph & 45. Civic \\
2. Ethereum & 13. Tron & 24. FTX Token & 35. Bittorrent & 46. Ankr \\
3. Ripple (XRP) & 14. Tezos & 25. Blockstack & 36. Nano & 47. iExec RLC \\
4. Litecoin & 15. Cosmos & 26. OMG Network & 37. Enjin Coin & 48. Tomochain \\
5. Bitcoin Cash & 16. VeChain & 27. Ontology & 38. Band Protocol & 49. Fantom \\
6. Binance Coin & 17. NEO & 28. Algorand & 39. Chilliz & 50. Pundi X \\
7. Cardano & 18. Ziliqa & 29. Basic Attention Token & 40. Holo & 51. Wanchain \\
8. Chainlink & 19. Dash & 30. REN & 41. IOST & 52. IoTeX \\
9. Stellar & 20. Dogecoin & 31. OX & 42. Ravencoin & 53. Harmony \\
10. EOS & 21. Ethereum Classic & 32. ICON & 43. Matic Network & 54. Fetch.ai \\
11. Monero & 22. Zcash & 33. Qtum & 44. Kava.io & 55. FunFair
\end{tabular}


The n:m coherence and phase of price changes were computed pairwise for all the 55 cryptocurrencies. Both iso- and cross-frequency coupling (IFC and CFC) were detected as shown by the sample case of Bitcoin vs. Litecoin in Figure 1.
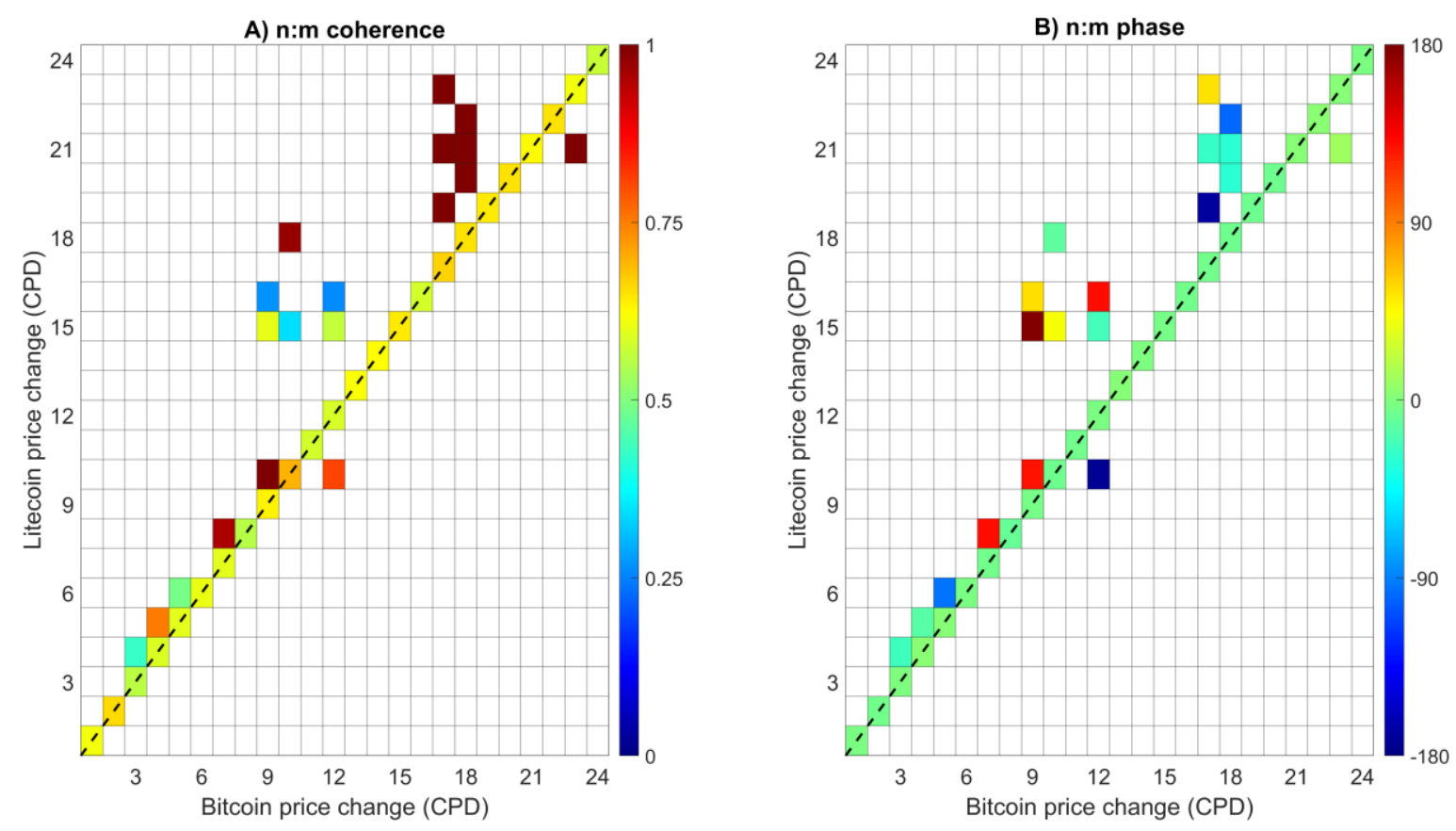

Fig. 1A. n:m coherence between intra-day price changes of Bitcoin vs. Litecoin. Both isofrequency $(1: 1)$ and cross-frequency coupling $(m: n$, where $m \neq n)$ were detected. Cross frequency coupling includes both integer and non-integer harmonic $(m>n)$ and sub-harmonic $(\mathrm{m}<\mathrm{n})$ coupling. Thus, harmonic and sub-harmonic coupling includes all the coupling values above and below the iso-frequency coupling respectively (indicated by the black dashed diagonal line). Coherence values below the significance level are rendered 0 and omitted for clarity. 1B. n:m phase between intra-day price changes of Bitcoin vs. Ethereum. Phase values are in degrees and range from -180 to +180 . [CPD $=$ cycles per day]

For every pair of cryptocurrencies, the sum of significant (i) IFC, (ii) harmonic CFC, and (iii) sub-harmonic CFC were computed along with their mean phases. Figure 2 depicts the total IFC and corresponding mean phase for each pair of cryptocurrencies. Ethereum was found to have the highest mean IFC (mean \pm std $=9.90 \pm 4.16$ ) while Ankr had the lowest mean IFC (mean \pm std $=1.40 \pm 3.16$ ). Furthermore, correlation analysis revealed significant negative relationships between the market capital rank and (i) the mean IFC value (i.e., the mean of the total IFC of each cryptocurrency $i$ with all other cryptocurrencies, $\rho=-0.69, \mathrm{p}<0.001$; Fig. $3 \mathrm{~A}$ ), (ii) the grand mean of the IFC phase (i.e., the average of the mean IFC phase of each cryptocurrency $i$ with all other cryptocurrencies, $\rho=-0.74, \mathrm{p}<0.001$; Fig. 3B). Nevertheless, there were certain exceptions to this generalization. For example, it can be seen in Figure 2 that Theta network had relatively low IFC with other cryptocurrencies despite having a large market 
capitalization. On the other hand, IOST, Ravencoin and Matic network had high IFC with other cryptocurrencies while having low market capitalization.

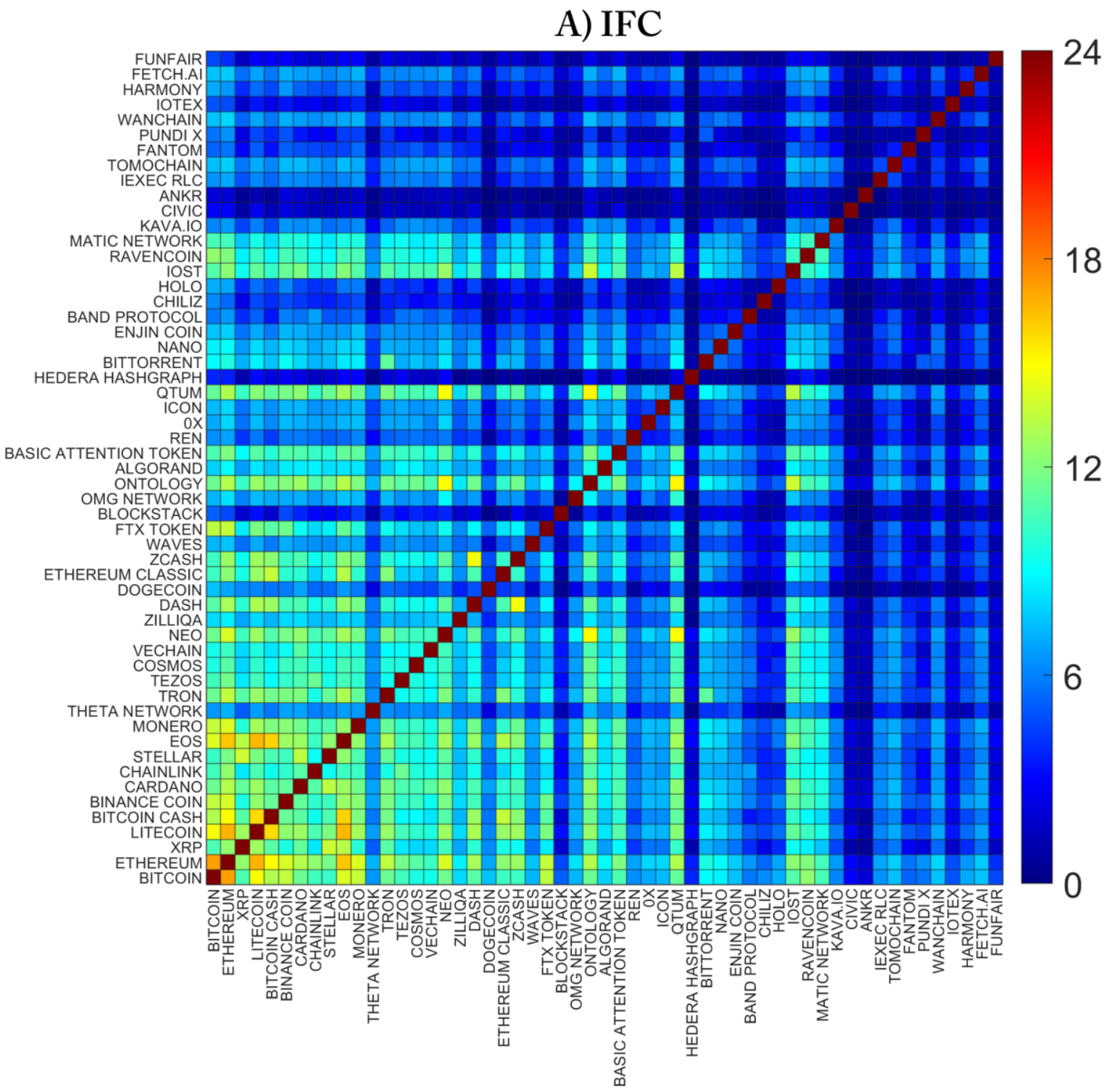

Fig. 2A. Total pairwise iso-frequency coupling (IFC). Each pixel value corresponds to the sum of all significant IFC values for each pair of cryptocurrencies. 


\section{B) IFP}

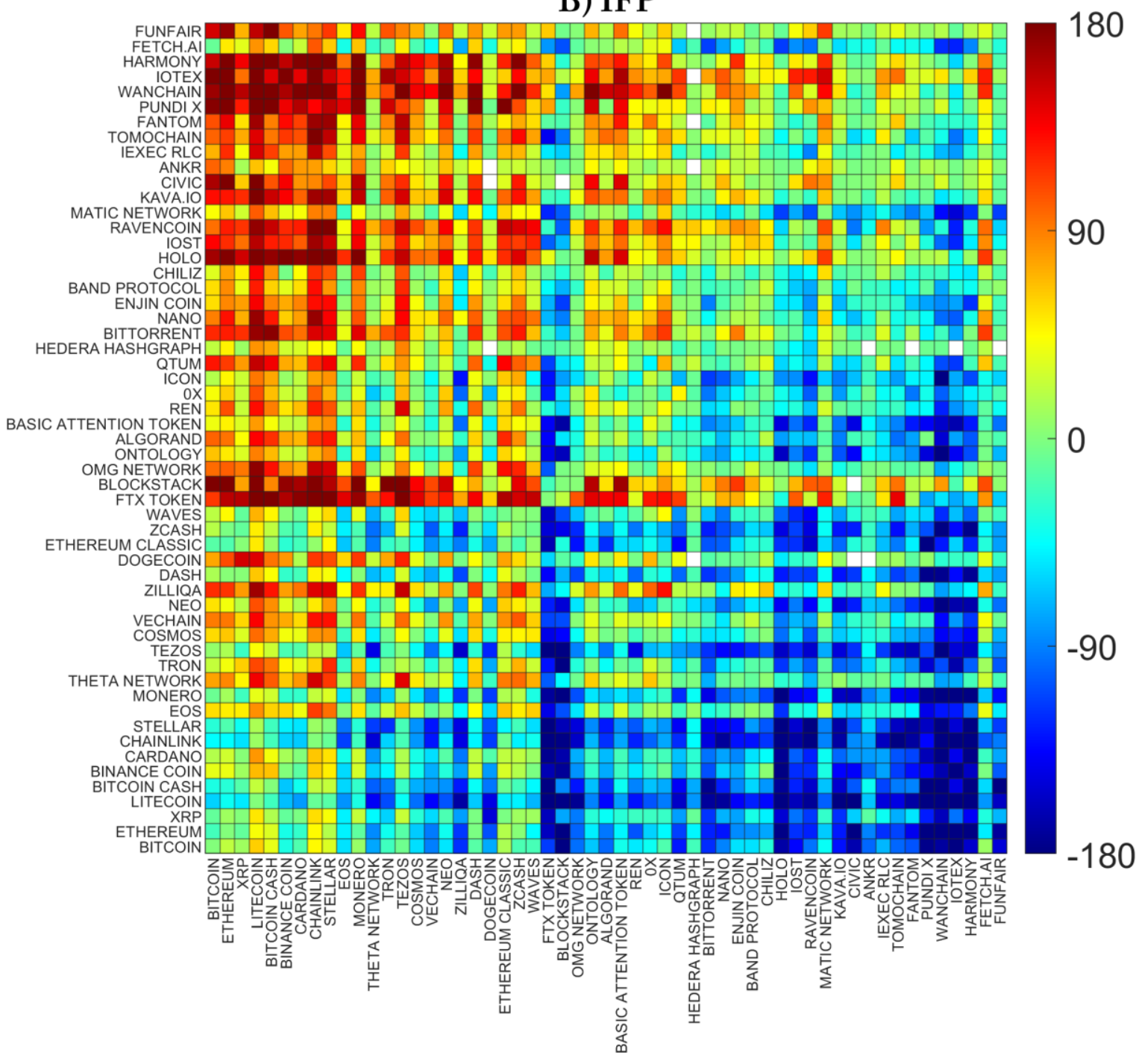

Fig. 2B. Mean pairwise iso-frequency coupling phase (IFP). Each pixel value corresponds to the mean of the phases of all significant IFC values for each pair of cryptocurrencies. Phase values are in degrees and range from -180 to +180 . 

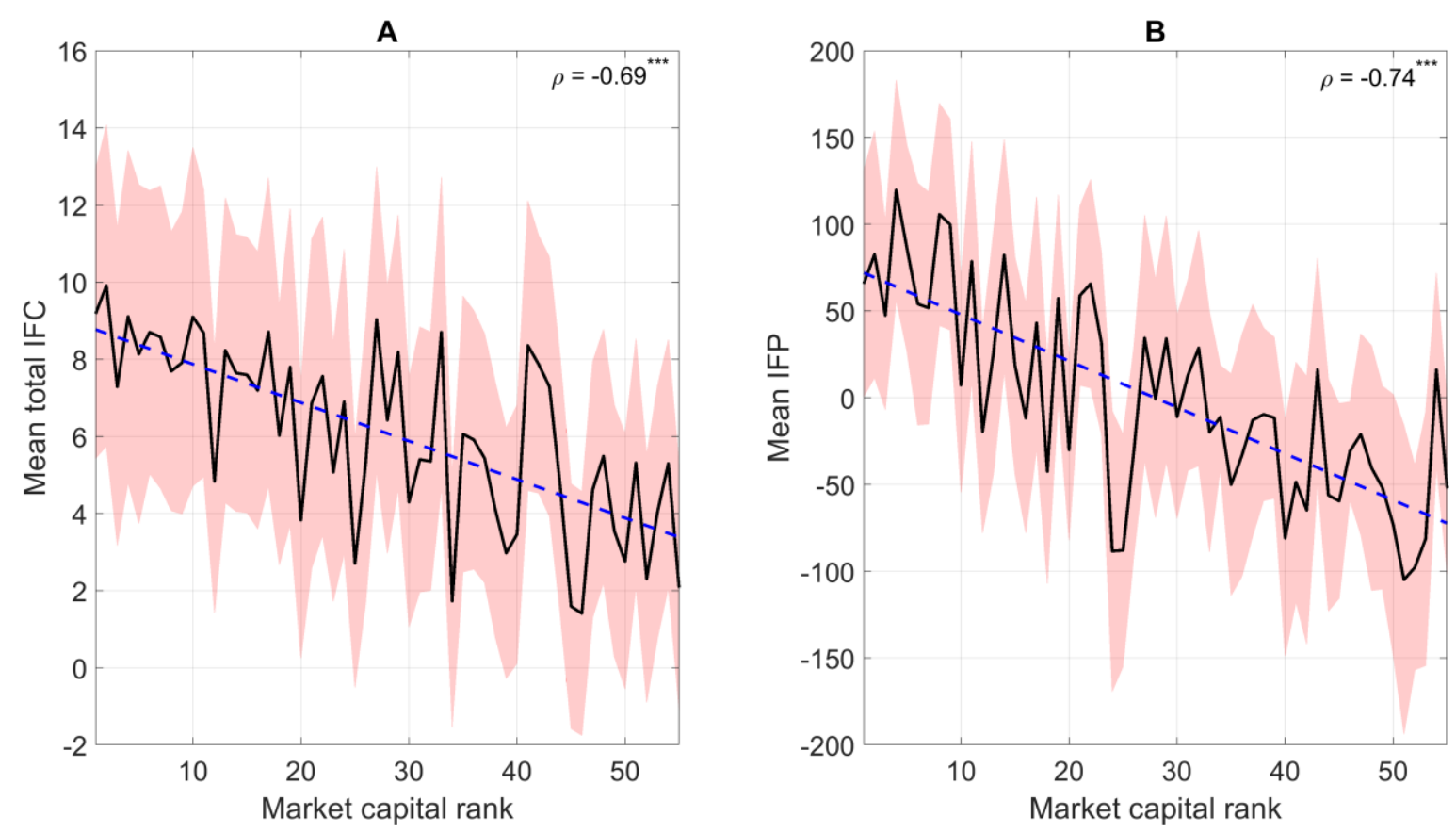

Fig 3A. Total iso-frequency coupling (IFC) vs. market capital rank. The total IFC mean \pm std for each cryptocurrency along with the linear fit to the mean curve (mean total IFC $=-0.1 \times$ market capital rank +8.8 ) is shown. 3B. Mean iso-frequency coupling phase (IFP) vs. market capital rank. The grand mean \pm std of IFC phases for each cryptocurrency along with the linear fit to the mean curve (grand mean IFP $=-2.7 \times$ market capital rank +74.7 ) is shown. Phase values are in degrees and range from -180 to +180 . (mean \pm std $=$ black curve \pm red shaded region; linear fit $=$ blue dashed line; $* * * \mathrm{p}<0.001)$

Figure 4 depicts the total CFC and corresponding mean phase for each pair of cryptocurrencies. Since coherence is a symmetrical measure, depending on whether the values are read as Y vs. X or X vs. Y, they depict the total amount of subharmonic or harmonic CFC, i.e., the sum of all values below or above the main diagonal as shown in Figure 1, respectively. Moreover, by comparing Figure 2A with Figure 4A it can be seen that iso-frequency coupling is symmetric while cross-frequency coupling is antisymmetric about the main diagonal. Fetch.ai was found to have the highest mean subharmonic CFC (mean $\pm \mathrm{std}=5.3 \pm 3.1$ ) while Dogecoin had the lowest mean subharmonic CFC (mean $\pm \mathrm{std}=1.40 \pm 3.16)$. On the other hand, Binance coin had the highest mean harmonic CFC (mean \pm std $=6.9 \pm 2.7)$ while FTX token had the lowest mean harmonic CFC (mean \pm std $=2.7 \pm 0.6$ ). Unlike in the case of IFC, no significant correlations were found between the market capital rank and (i) the mean subharmonic CFC value (i.e., the mean of the total subharmonic CFC of each cryptocurrency $i$ with all other cryptocurrencies, $\rho=0.10, \mathrm{p}=0.48$; Fig. $5 \mathrm{~A}$ ), or (ii) the grand mean of the CFC phase (i.e., the average of the mean subharmonic CFC phase of each cryptocurrency $i$ with all other cryptocurrencies, $\rho=0.14, \mathrm{p}=0.31$; Fig. 5B). Likewise, there was a weak negative correlation between the market capital rank and (i) the mean harmonic CFC value (i.e., the mean of the total harmonic CFC of each cryptocurrency $i$ with all other cryptocurrencies, $\rho=-0.33, \mathrm{p}=$ 0.01 ; Fig. 5C), but no significant correlation between the market capital rank and the grand 
mean of the harmonic CFC phase (i.e., the average of the mean harmonic CFC phase of each cryptocurrency $i$ with all other cryptocurrencies, $\rho=-0.08, \mathrm{p}=0.57$; Fig. 5D).

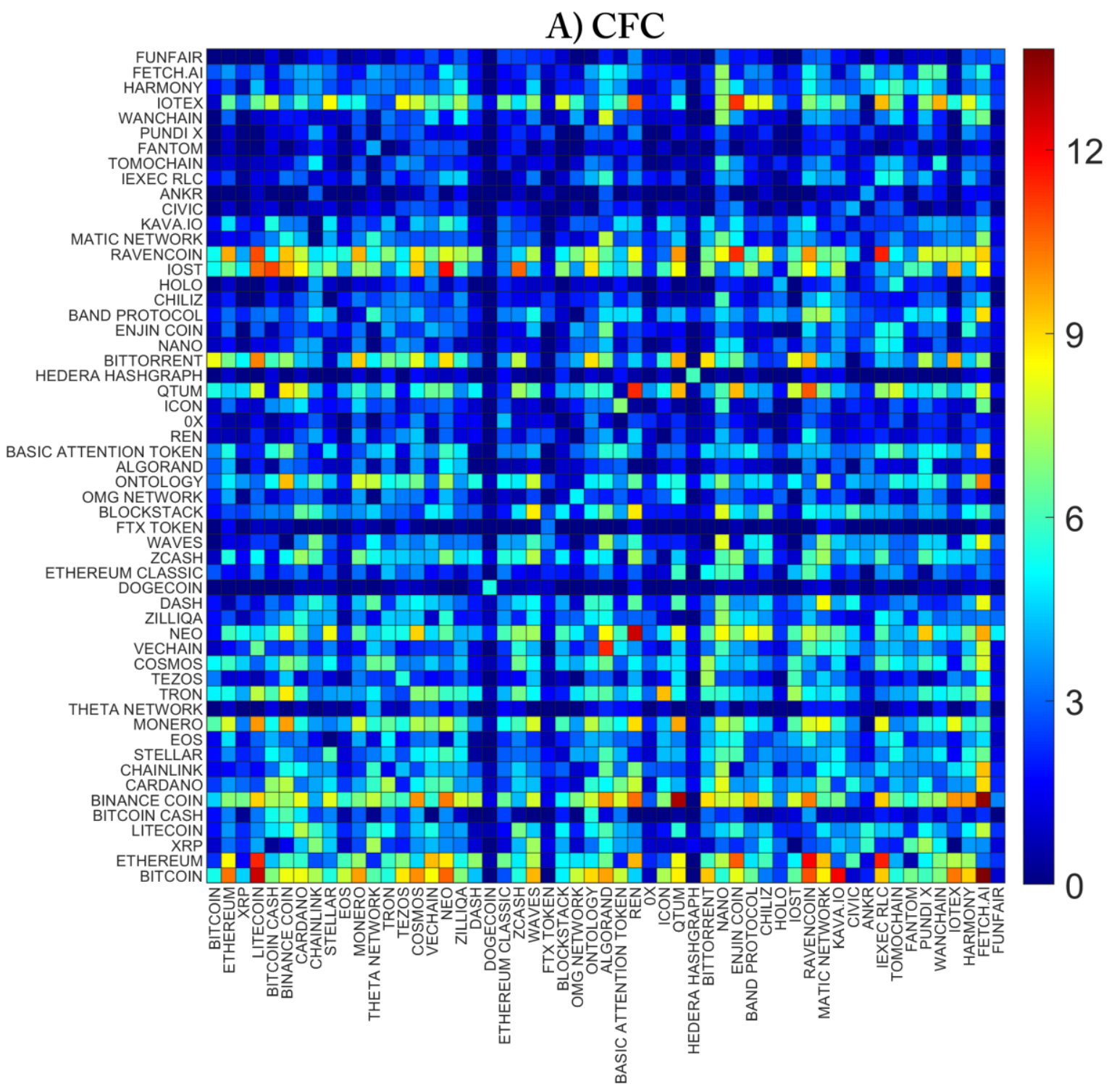

Fig. 4A. Total pair-wise cross-frequency coupling (CFC). Each pixel value corresponds to the sum of all significant $\mathrm{CFC}$ values for each pair of cryptocurrencies. 


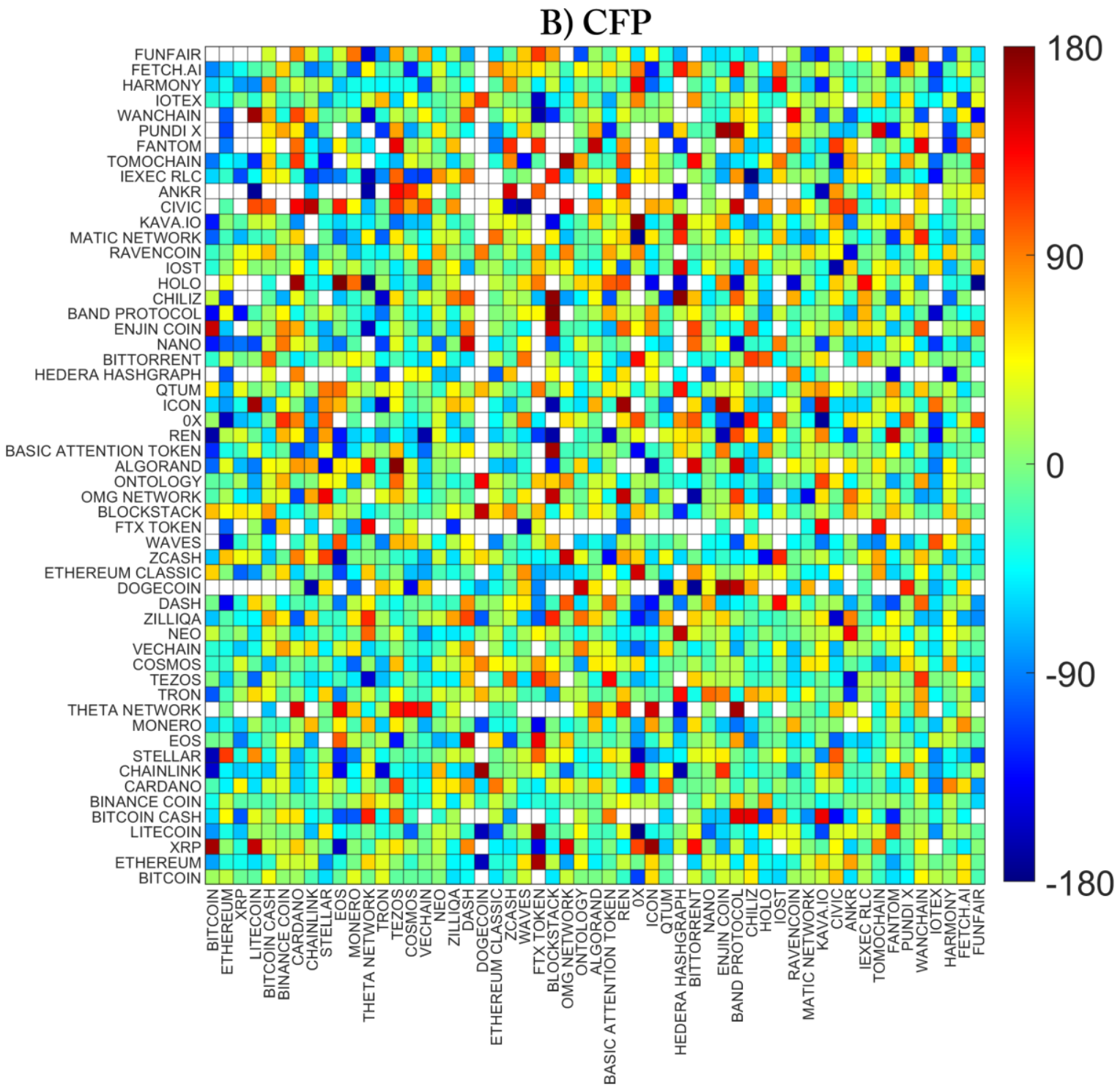

Fig. 4B. Mean pair-wise cross-frequency coupling phase (CFP). Each pixel value corresponds to the mean of the phases of all significant CFC values for each pair of cryptocurrencies. Phase values are in degrees and range from -180 to +180 . (X:Y $=$ subharmonic coupling; $\mathrm{Y}: \mathrm{X}=$ harmonic coupling) 

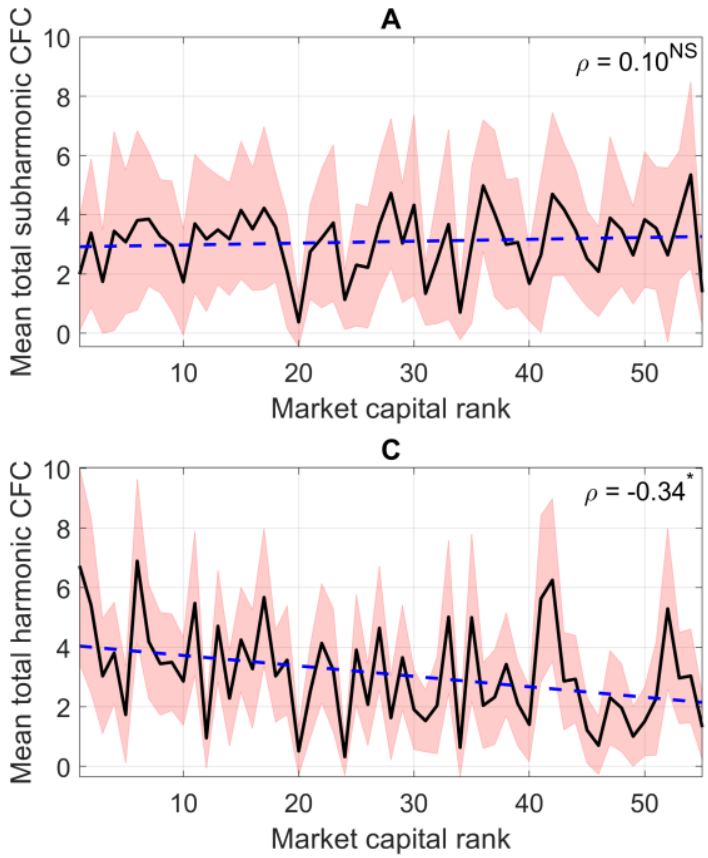

B
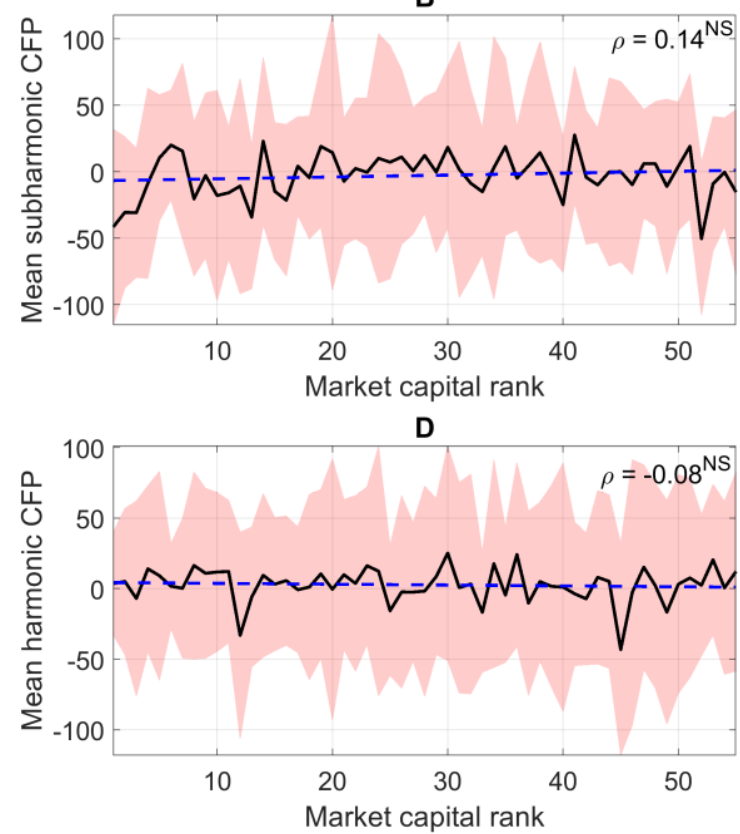

Fig. 5A. Total subharmonic cross-frequency coupling (CFC) vs. market capital rank. The total subharmonic CFC mean \pm std for each cryptocurrency along with the linear fit to the mean curve (mean total subharmonic $\mathrm{CFC}=0.006 \times$ market capital rank +2.9 ) is shown. 5B. Mean subharmonic cross-frequency coupling phase (CFP) vs. market capital rank. The grand mean \pm std of subharmonic CFC phases for each cryptocurrency along with the linear fit to the mean curve (grand mean subharmonic CFP $=0.14 \times$ market capital rank -6.74 ) is shown. 5C. Total harmonic cross-frequency coupling (CFC) vs. market capital rank. The total harmonic $\mathrm{CFC}$ mean \pm std for each cryptocurrency along with the linear fit to the mean curve (mean total harmonic $\mathrm{CFC}=-0.03 \times$ market capital rank +4.0 ) is shown. 5D. Mean harmonic cross-frequency coupling phase (CFP) vs. market capital rank. The grand mean \pm std of harmonic $\mathrm{CFC}$ phases for each cryptocurrency along with the linear fit to the mean curve (grand mean harmonic $\mathrm{CFP}=-0.06 \times$ market capital rank +4.33$)$ is shown. Phase values are in degrees and range from -180 to +180 . (mean \pm std $=$ black curve \pm red shaded region; linear fit $=$ blue dashed line; NS $=$ not significant, $\mathrm{p}>0.05 ;{ }^{*} \mathrm{p}<=0.05$ ). 
Next, the MATLAB function linkage was used to construct a binary cluster tree/dendrogram to visualize the multilevel hierarchical clustering of various cryptocurrencies based on a distance metric calculated from the pair-wise total IFC. The total IFC was normalized by its theoretical maximum $(=24)$ for this purpose. The dendrogram obtained by the 'average' algorithm of the linkage function was found to have the highest cophenetic correlation coefficient $(\rho=0.95)$. Figure 6 depicts this dendrogram. Some cryptocurrencies with common features formed identifiable clusters (link height $<0.5$ ): (i) Bitcoin and its forks Litecoin and Bitcoin Cash, ${ }^{38,39}$ along with the smart-contract enabled cryptocurrencies Ethereum and EOS, ${ }^{40}$ (ii) NEO, Ontology and Qtum, all of which have proof-of-stake based consensus algorithms and were developed in Asia, ${ }^{41}$ (iii) Privacy-centered cryptocurrencies Dash and Zcash, ${ }^{42}$ and (iv) Ripple and Stellar, both of which have a common founder and are marketed as decentralized payment networks. ${ }^{43}$ On the other hand, some of the clustering is a consequence of investment strategies associated with market capitalization rather than fundamentals. This is clearly visible from the shorter distance between Bitcoin and Ethereum as compared to that of Bitcoin Cash despite the latter being a direct fork of Bitcoin. ${ }^{44}$ Nevertheless, most of the links in the dendrogram were found to be consistent i.e., approximately the same height as the links below them (excluding any leaf node). This shows the absence of any major natural division in the data. $^{45-47}$

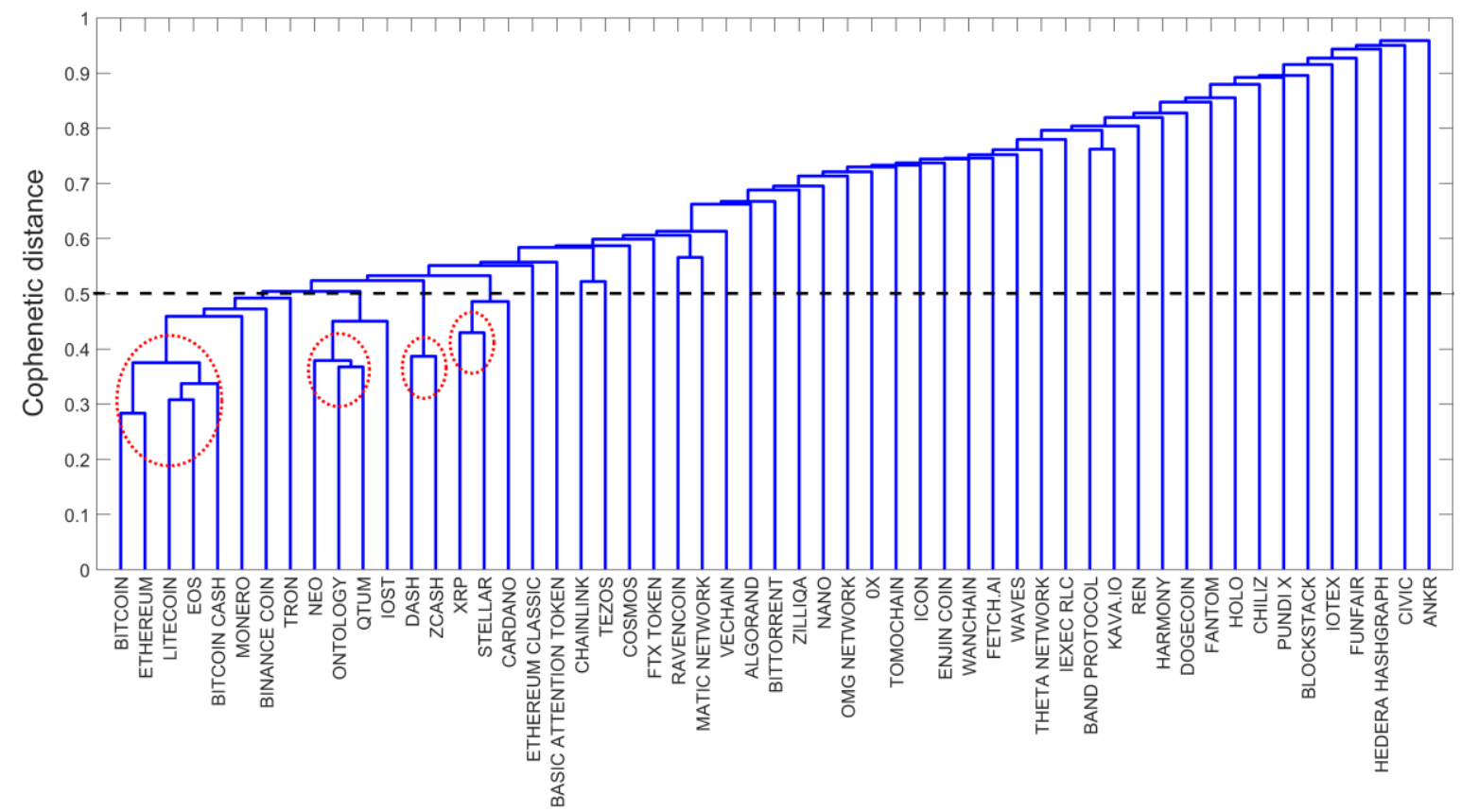

Fig. 6. IFC based dendrogram. The dendrogram/binary cluster tree was constructed using a distance metric $\mathrm{D}_{\mathrm{ij}}=1-\mathrm{IFC}_{\mathrm{ij}}$ for each pair of cryptocurrencies $i$ and $j$ where IFC is the normalized total iso-frequency coupling between them. The height (cophenetic distance) of a link in this dendrogram represents the distance between the corresponding two clusters that contain any two cryptocurrencies. Visually identifiable clusters with link height $<0.5$ are demarcated by red circles. 
Finally, the risk reduction associated with diversification of portfolio between two cryptocurrencies $(\mathrm{X}, \mathrm{Y})$ as compared to a portfolio having only the larger market capital cryptocurrency (X) was assessed for each pair of cryptocurrencies. Figure 7A shows the total risk reduction achieved by portfolio diversification among every pair of cryptocurrencies for synchronous trading conditions (i.e., same trading frequency for both cryptocurrencies in the pair). Risk reduction is best achieved by pairing those smaller market capital cryptocurrencies (e.g., Theta network, Dogecoin and Hedera Hashgraph) which have low IFC with the larger market capital cryptocurrencies. Likewise, pairing of smaller market capital cryptocurrencies (e.g., Matic network and IOST) which have high IFC with the larger market capital cryptocurrencies can lead to increased risk. Overall, however, weak negative correlation was found between the mean total IFC and risk reduction for synchronous trading frequencies $(\rho=$ $-0.37, \mathrm{p}<0.001)$. Figures $7 \mathrm{~B}$ and $7 \mathrm{C}$ show the total risk reduction achieved by portfolio diversification for asynchronous trading conditions (i.e., trading frequencies for the two cryptocurrencies in the pair are different). When the larger market capital cryptocurrency is traded at a higher frequency (Fig. 7B), risk reduction is achieved by portfolio diversification for almost all pairs of cryptocurrencies (mean $\pm s t d=2.36 \pm 1.74$ ). A mild negative correlation was found between the amount of mean total risk reduction and the market capital rank of the larger market capital cryptocurrency, but it was not statistically significant $(\rho=-0.23, \mathrm{p}=0.09)$. On the other hand, when the larger market capital cryptocurrency is traded at a lower frequency (Fig. 7C), the benefit of portfolio diversification is confined to high market capital cryptocurrencies like Bitcoin, Ethereum and Binance coin. For lower ranked cryptocurrencies, there is an increase in risk as in the case of Qtum, Ontology, Chilliz and Civic. There are, however, exceptions to this, as in the case of Ripple and Bitcoin cash, which, despite being high ranked, show increase in risk whereas Ravencoin, IOST and Matic Network show risk reduction despite being low ranked. Overall, there was a mild negative correlation was found between the amount of mean total risk reduction and the market capital rank of the larger market capital cryptocurrency, but it was not statistically significant $(\rho=-0.22, \mathrm{p}=0.09)$. 


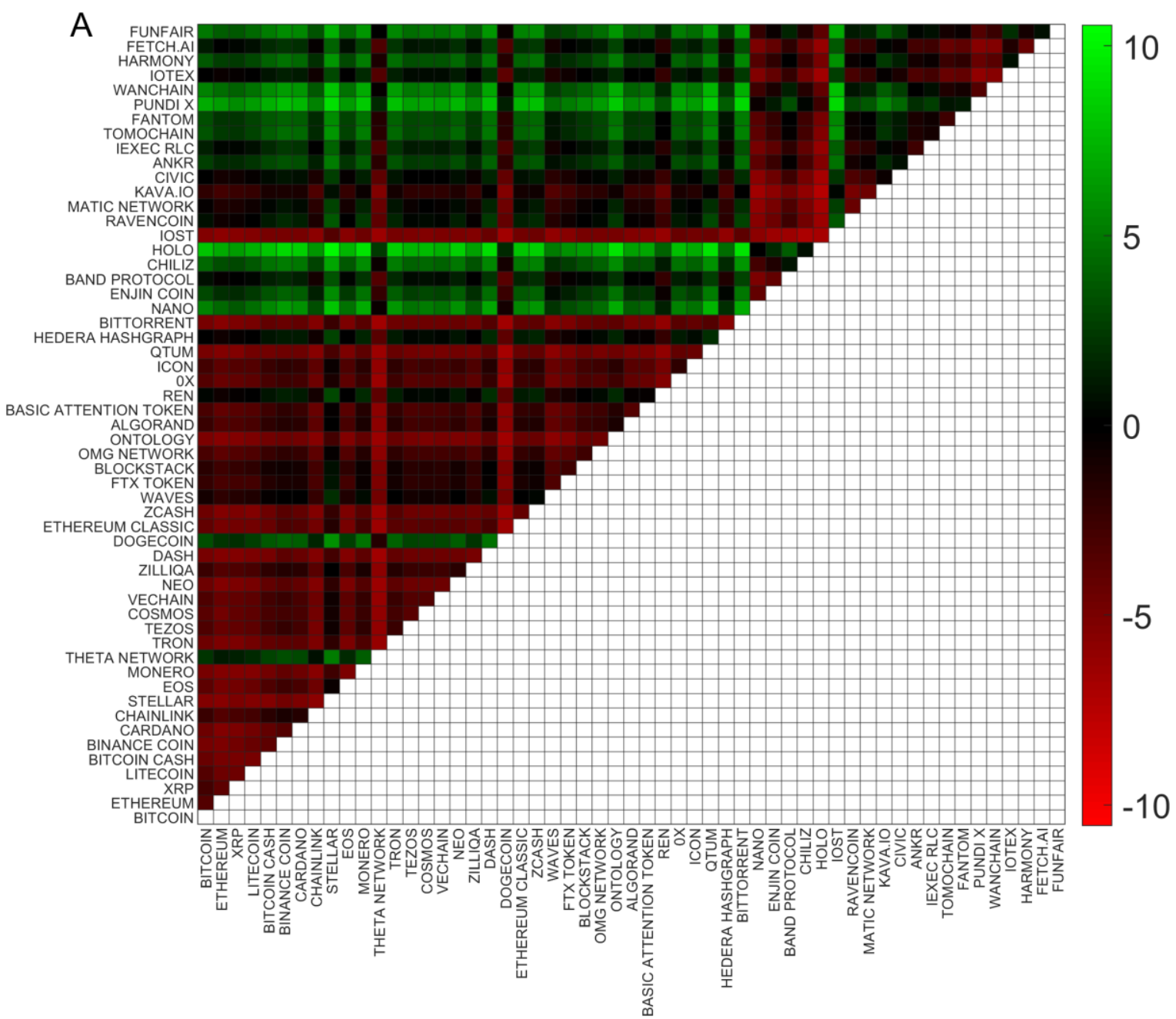

Fig. 7. Risk reduction. 7A. Synchronous trading condition. Each pixel value corresponds to the total risk reduction achieved by diversification of portfolio between the corresponding pair of cryptocurrencies for all synchronous trading frequencies (1-24 Hz). Positive values indicate net reduction of risk while negative values indicate net increase of risk as compared to a portfolio containing only the cryptocurrency with the higher market capital in the pair. 


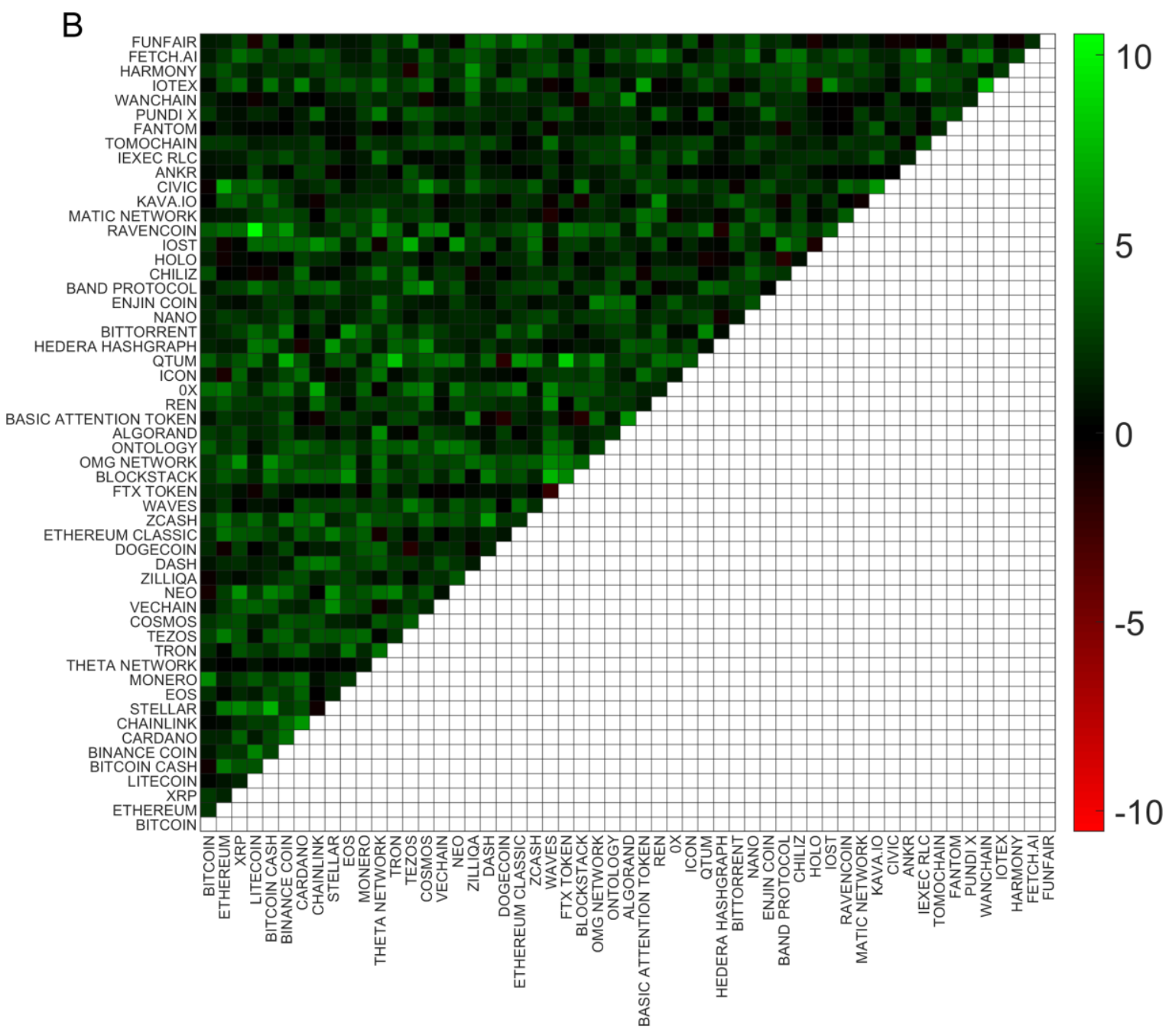

Fig. 7B. Asynchronous trading condition with lower frequency of trading for the smaller market capital cryptocurrency. Pixel values mean the same as in $7 \mathrm{~A}$, except that the larger market capital cryptocurrency in the corresponding pair is traded at a lower frequency. 


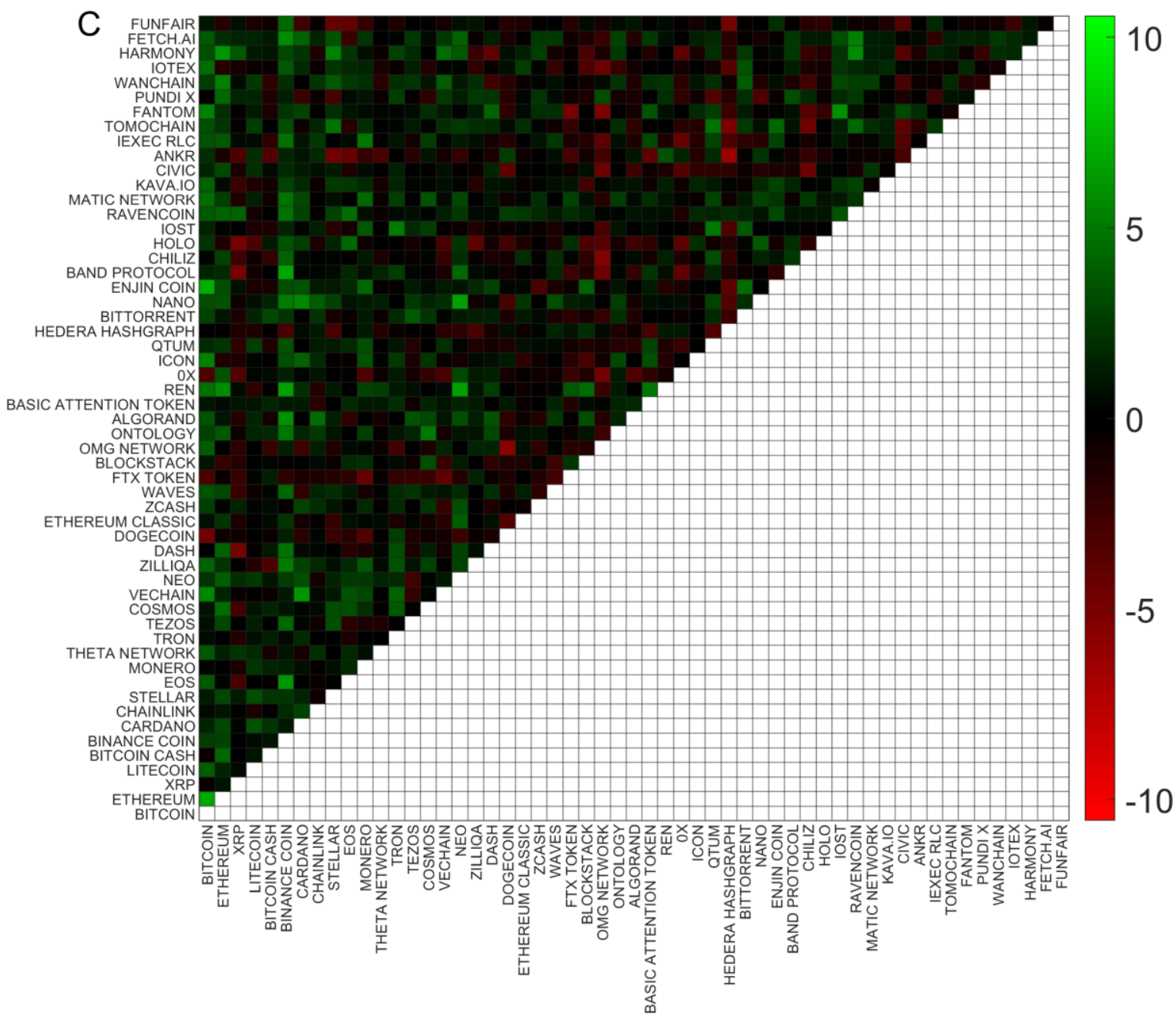

Fig. 7C. Asynchronous trading condition with higher frequency of trading for the smaller market capital cryptocurrency. Pixel values mean the same as in 7A, except that the larger market capital cryptocurrency in the corresponding pair is traded at a higher frequency.

\section{Discussion and Conclusion}

In this study, the pairwise relationship between the intra-day price changes of cryptocurrencies for 2020 were studied using a generalized coherence measure to quantify iso-frequency (IFC) and cross-frequency (CFC) coupling between them. The amount of total mean IFC as well as its mean phase was found to decrease with decrease in market capital size, showing that smaller market capital cryptocurrencies exhibit more independent price movements. Certain cryptocurrencies like Theta network show low mean total IFC despite having high market capital. Such assets may serve to act as a safe hedge when price changes of other large market 
capital cryptocurrencies show herding behavior due to the development of a price bubble. Likewise, certain low market capital cryptocurrencies like Ravencoin that show high mean IFC with the large market capital cryptocurrencies can serve as a good investment alternative for those who wish to avoid the high transaction fees associated with top cryptocurrencies like Bitcoin or Ethereum. Furthermore, using hierarchical cluster analysis of the IFC values, certain functionally distinct clusters of cryptocurrencies were found based on their hashing algorithm, development history and proposed applications of use. This reflects common investment goals and perspectives for traders investing in closely related cryptocurrencies.

Unlike the IFC, the mean total subharmonic CFC and phase was not found to have any relationship with the market capital size of the cryptocurrencies. Similarly, the mean total harmonic CFC had only a weak negative correlation with market capital size while the former's phase had no correlation with the latter. This shows that for asynchronous trading frequencies, the cryptocurrency market is much more diversified and does not show herding behavior. From an ecosystem point of view, this lends credibility to the fact that market capital driven investment strategy lends less importance to asset fundamentals. Thus, at least some of the panic driven flash crashes that currently plague the cryptocurrency markets maybe be indicative of an inefficient market rather than inevitable correction of price bubbles. ${ }^{48}$

The risk reduction associated with portfolio diversification between a small and a large market capital cryptocurrency was assessed for both synchronous and asynchronous trading frequencies of both. Diversifying portfolios to include smaller market capital cryptocurrencies which have price movements independent of the larger market capital cryptocurrency showed positive risk reduction for synchronous trading conditions. In asynchronous trading conditions, when the larger market capital cryptocurrency is traded at a relatively higher frequency, portfolio diversification to a smaller market capital cryptocurrency almost always leads to reduced risk irrespective of the degree of price coherency between them. In other words, it entails a strategy of short-term trading of well-established assets while 'HODL'ing newly emerging ones. This, we believe, is a hallmark of an emergent market still in its 'discovery' phase wherein many new asset classes (each with its own new potential niche of use cases) are rising every day.

When the larger market capital cryptocurrency is traded at a relatively lower frequency, however, portfolio diversification may lead to either reduced or increased risk depending upon the pair of cryptocurrencies being considered.

There are a few limitations of this study. The data for this study was obtained from a single centralized exchange (Binance). However, considering its high 24-hour trading volume and reputation among the cryptocurrency trading community, ${ }^{49}$ it is unlikely that the overall results of this study will not be generalizable to other trading platforms. Due to consideration of the entire period of the year 2020, most of the decentralized finance-based tokens could not be included in this study. Hence, it may be interesting to investigate such tokens using the approach of this study as more data is generated in the future. This study was confined to investigation of the intra-day price changes only. For investigating longer periods, the statistical power of the generalized coherence analysis is limited by the availability of the number of epochs of data. For example, since cryptocurrency trading data is mostly available for the past 10 years or so, for investigation of intra-month price changes only around 120 epochs (12 months per year $\times$ 10 years) are available so far. This might lead to a reduced power of analysis. ${ }^{50}$ Moreover, given 
the poor survivability of most cryptocurrencies over such long periods of time, ${ }^{51}$ such an analysis might not be meaningful at all.

\section{Author Contributions}

N.S. conceived this research work, collected the data, performed all the analyses, and drafted the manuscript. N.S. and Y.Y. revised the manuscript and drafted the response to reviewers.

\section{Conflict of Interest}

The authors declare no financial or any other conflicts of interest.

\section{Notes and Reference}

${ }^{1}$ Nakamoto, S. "Bitcoin: A Peer-to-Peer Electronic Cash System.” (accessed 31 December 2020) https://bitcoin.org/bitcoin.pdf.

${ }^{2}$ Nica, O. Piotrowska, K, Schenk-Hoppé, K. R. "Cryptocurrencies: Economic Benefits and Risks." European Economics: Macroeconomics \& Monetary Economics eJournal, 2017.

${ }^{3}$ Chen, Y., Bellavitis, C. "Blockchain Disruption and Decentralized Finance: The Rise of Decentralized Business Models.” Journal of Business Venturing Insights 13 e00151 (2020) https://doi.org/10.1016/j.jbvi.2019.e00151.

${ }^{4}$ Weber, B. "Bitcoin and the Legitimacy Crisis of Money." Cambridge Journal of Economics 40.1 17-41 (2016) http://doi.org/10.1093/cje/beu067.

5 Yermack, D. “Chapter 2 - Is Bitcoin a Real Currency? An Economic Appraisal.” In Lee Kuo Chuen, D., Ed., Handbook of Digital Currency. San Diego: Academic Press (2015) 31-43.

${ }^{6}$ Bonneau, J., Miller, A., Clark, J., Narayanan, A., Kroll, J. A., Felten, E. W. "SoK: Research Perspectives and Challenges for Bitcoin and Cryptocurrencies." In 2015 IEEE Symposium on Security and Privacy, 17-21 May 2015 104-121 (2015) https: / / doi .org/10.1109/SP.2015.14.

${ }^{7}$ Kethineni, S., Cao, Y. "The Rise in Popularity of Cryptocurrency and Associated Criminal Activity." International Criminal Justice Review 30.3 325-344 (2019) https://doi.org/10.1177/1057567719827051.

${ }^{8}$ Glaser, F., Zimmermann, K., Haferkorn, M., Weber, M. C. 'Bitcoin - Asset or Currency? Revealing Users' Hidden Intentions." SSRN (accessed 20 September 2021) https : / ssrn . com/abstract=2425247.

${ }^{9}$ Enoksen, F. A., Landsnes, C. J., Lučivjanská, K., Molnár, P. “Understanding Risk of Bubbles in Cryptocurrencies.” Journal of Economic Behavior \& Organization 176 129-144 (2020)

https://doi.org/10.1016/j.jebo.2020.05.005.

${ }^{10}$ CoinMarketCap. “Today’s Cryptocurrency Prices by Market Cap.” (accessed 12 December 2020) https: / / coinmarketcap.com/.

${ }^{11}$ ElBahrawy, A., Alessandretti, L., Kandler, A., Pastor-Satorras, R., Baronchelli, A. "Evolutionary Dynamics of the Cryptocurrency Market." Royal Society Open Science 4.11 170623

https://doi.org/10.1098/rsos.170623. 
${ }^{12}$ Lucchini, L., Alessandretti, L., Lepri, B., Gallo, A., Baronchelli, A. "From Code to Market: Network of Developers and Correlated Returns of Cryptocurrencies." Science Advances 6.51 eabd2204 (2020) https://doi.org/10.1126/sciadv.abd2204.

${ }^{13}$ Sigaki, H. Y. D., Perc, M., Ribeiro, H. V. "Clustering Patterns in Efficiency and the Coming-of-Age of the Cryptocurrency Market." Scientific Reports 9.11440 (2019) https://doi.org/10.1038/s41598-01837773-3.

${ }^{14}$ Bouri, E., Gupta, R., Roubaud, D. "Herding Behaviour in Cryptocurrencies." Finance Research Letters 29 216-221 (2019) https://doi.org/10.1016/j.frl.2018.07.008.

${ }^{15}$ Stosic, D., Stosic, D., Ludermir, T. B., Stosic, T. "Collective Behavior of Cryptocurrency Price Changes." Physica A: Statistical Mechanics and its Applications 507 499-509 (2018)

https://doi.org/10.1016/j.physa.2018.05.050.

${ }^{16}$ Gao, B., et al. "Tracking Counterfeit Cryptocurrency End-to-End," Proc. ACM Meas. Anal. Comput. Syst. 4.3 Article 50 (2020) https: / / doi.org/10.1145/3428335.

${ }^{17}$ Liu, W. "Portfolio Diversification Across Cryptocurrencies." Finance Research Letters 29 200-205 (2019) https://doi.org/10.1016/j.frl.2018.07.010.

${ }^{18}$ Plerou, V., Gopikrishnan, P., Rosenow, B., Nunes Amaral, L. A., Stanley, H. E. "Universal and Nonuniversal Properties of Cross Correlations in Financial Time Series." Physical Review Letters 83.7 1471-1474 (1999) https://doi.org/10.1103/PhysRevLett.83.1471.

${ }^{19}$ Cai, X. J., Tian, S., Yuan, N., Hamori, S. "Interdependence Between Oil and East Asian Stock Markets: Evidence from Wavelet Coherence Analysis." Journal of International Financial Markets, Institutions and Money 48 206-223 (2017) https://doi.org/10.1016/j.intfin.2017.02.001.

${ }^{20}$ Katsiampa, P., Corbet, S., Lucey, B. "High Frequency Volatility Co-Movements in Cryptocurrency Markets." Journal of International Financial Markets, Institutions and Money 62 35-52 (2019) https://doi.org/10.1016/j.intfin.2019.05.003.

${ }^{21}$ Katsiampa, P., Corbet, S., Lucey, B. "Volatility Spillover Effects in Leading Cryptocurrencies: A BEKKMGARCH Analysis." Finance Research Letters 29 68-74 (2019)

https://doi.org/10.1016/j.frl.2019.03.009.

${ }^{22}$ Qiao, X., Zhu, H., Hau, L. "Time-Frequency Co-Movement of Cryptocurrency Return and Volatility: Evidence from Wavelet Coherence Analysis." International Review of Financial Analysis 71101541 (2020) https://doi.org/10.1016/j.irfa.2020.101541.

${ }^{23}$ Aguiar-Conraria, L., Azevedo, N., Soares, M. J. "Using Wavelets to Decompose the Time-Frequency Effects of Monetary Policy." Physica A: Statistical Mechanics and its Applications 387.12 2863-2878 (2008) https://doi.org/10.1016/j.physa.2008.01.063.

${ }^{24}$ Phillips, R. C., Gorse, D. “Cryptocurrency Price Drivers: Wavelet Coherence Analysis Revisited." PLOS ONE 13.4 e0195200 (2018) https://doi.org/10.1371/journal.pone.0195200.

${ }^{25}$ Omane-Adjepong, M., Alagidede, I. P. "Multiresolution Analysis and Spillovers of Major Cryptocurrency Markets." Research in International Business and Finance 49 191-206 (2019)

https://doi.org/10.1016/j.ribaf.2019.03.003.

${ }^{26}$ CoinGecko. "Cryptocurrency Prices by Market Cap.” (accessed 20 September 2021)

https://www.coingecko.com/en.

${ }^{27}$ McHardy, S. "python-binance (Version 0.7.7) [Source code]." (accessed 20 September 2021, current version 1.0.14) https://github.com/sammchardy/python-binance. 
${ }^{28}$ CoinGecko. "Top Cryptocurrency Exchanges Ranking by Trust Score - Spot." (accessed 20 September 2021) https://www.coingecko.com/en/exchanges.

${ }^{29}$ Yang, Y., Solis-Escalante,T. van der Helm, F. C., Schouten, A. C. "A Generalized Coherence Framework for Detecting and Characterizing Nonlinear Interactions in the Nervous System." IEEE Transactions on Biomedical Engineering 63.12 2629-2637 (2016) https://doi.org/10.1109/tbme.2016.2585097.

${ }^{30}$ Nikias, C. L., Mendel, J. M. "Signal Processing with Higher-Order Spectra." IEEE Signal Processing Magazine 10.3 10-37 (1993) https: / doi.org/10.1109/79.221324.

${ }^{31}$ Yang, Y., Solis-Escalante, T., van de Ruit, M., van der Helm, F. C. T., Schouten, A. C., "Nonlinear Coupling Between Cortical Oscillations and Muscle Activity During Isotonic Wrist Flexion." Frontiers in Computational Neuroscience 2016.10126 (2016) https://doi.org/10.3389/fncom.2016.00126.

${ }^{32}$ Yang, Y., Schouten, A. C., Solis-Escalante, T., van der Helm, F. C. "Probing the Nonlinearity in Neural Systems Using Cross-Frequency Coherence Framework." IFAC-PapersOnLine 48.28 1386-1390 (2015) https://doi.org/10.1016/j.ifacol.2015.12.326.

${ }^{33}$ De Hemptinne, C., et al. "Exaggerated Phase-Amplitude Coupling in the Primary Motor Cortex in Parkinson Disease." Proceedings of the National Academy of Sciences 110.12 4780-4785 (2013) https://dx.doi.org/10.1073/pnas.1214546110.

${ }^{34}$ Mantegna, R. N. "Hierarchical Structure in Financial Markets." The European Physical Journal B Condensed Matter and Complex Systems 11.1 193-197 (1999)

https://doi.org/10.1007/s100510050929.

${ }^{35}$ MathWorks. Statistical and Machine Learning Toolbox. Natick, Massachusetts: United States (2020).

${ }^{36}$ Sokal, R. R., Rohlf, F. J. “The Comparison of Dendrograms by Objective Methods.” Taxon 11.2 33-40 (1962) https:/doi.org/10.2307/1217208.

${ }^{37}$ Reboredo, J. C., Rivera-Castro, M. A. "Gold and Exchange Rates: Downside Risk and Hedging at Different Investment Horizons." International Review of Economics \& Finance 34 267-279 (2014) https://doi.org/10.1016/j.iref.2014.07.005.

${ }^{38}$ McMillan, R. "Ex-Googler Gives the World a Better Bitcoin.” Wired (2013)

https://www.wired.com/2013/08/litecoin/.

${ }^{39}$ Frankenfield, J. "Bitcoin Cash Definition." Investopedia (2020)

https://www.investopedia.com/terms/b/bitcoin-cash.asp.

${ }^{40}$ CryptoSlate. "Smart Contracts Coins." (accessed 20 September 2021)

https://cryptoslate.com/cryptos/smart-contracts/.

${ }^{41}$ Martinez, A. "China-Based Cryptos on the Rise: NEO, Ontology, and Qtum Technical Analysis." (2019) https://cryptoslate.com/china-based-cryptos-on-the-rise-neo-ontology-and-qtumtechnical-analysis/.

${ }^{42}$ Seth, S. "6 Private Cryptocurrencies," Investopedia (2021)

https://www.investopedia.com/tech/five-most-private-cryptocurrencies/.

${ }^{43}$ Won, D. "XLM vs. XRP (Stellar vs. Ripple): The 2020 Full Comparison.” (2020)

https://www.exodus.io/blog/stellar-vs-ripple/.

${ }^{44}$ Kwon, Y., Kim, H., Shin, J., Kim, Y. "Bitcoin vs. Bitcoin Cash: Coexistence or Downfall of Bitcoin Cash?” In 2019 IEEE Symposium on Security and Privacy (SP), 19-23 May 2019 (2019) 935-951

https://doi.org/10.1109/SP.2019.00075. 
${ }^{45}$ Jain, A. K., Dubes, R. C. Algorithms for Clustering Data. Prentice-Hall (1988).

${ }^{46}$ Zahn, C. T. "Graph-Theoretical Methods for Detecting and Describing Gestalt Clusters." IEEE

Transactions on Computers C-20.1 68-86 (1971) https://doi.org/10.1109/T-C.1971.223083.

${ }^{47}$ Mahdavi-Damghani, B., Fraser, R., Howell, J., Halldorsson, J. "Cryptocurrency Sectorisation Through Clustering and Webscraping: Application to Systematic Trading." (preprint) (2021)

https://doi.org/10.13140/RG.2.2.13136.84488.

${ }^{48}$ Bouchaud, J.-P. “Economics Needs a Scientific Revolution.” Nature 455.7217 1181-1181 (2008) https://doi.org/10.1038/4551181a.

${ }^{49}$ Conway, L. "Best Crypto Exchanges." Investopedia. (2021) https: //www. investopedia.com/bestcrypto-exchanges-5071855.

50 Thompson, R. O. R. Y. “Coherence Significance Levels.” Journal of Atmospheric Sciences 36.102020 2021 (1979) https://doi.org/10.1175/1520-0469 (1979) 036<2020:CSL>2.0.CO;2.

${ }^{51}$ Lansky, J. "Practical Applications of Cryptocurrency Survival Analysis." The Journal of Alternative Investments 22.Supplement2 1 (2020) https://doi.org/10.3905/jai.22.s2.020.

(cc) BY ULSS 